Tinbergen Institute Discussion Paper
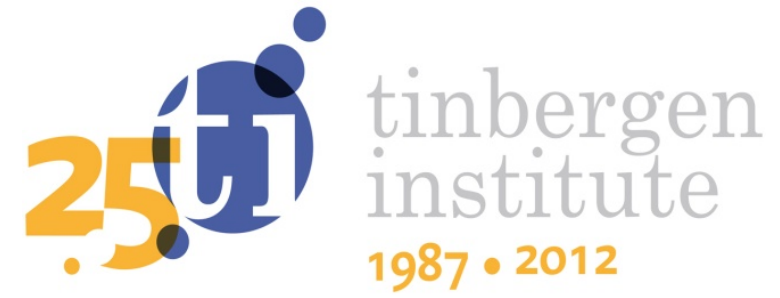

\title{
The Average Tree Permission Value for Games with a Permission Tree
}

\author{
Rene van den Brink' \\ Jean-Jacques Herings² \\ Gerard van der Laan' \\ Dolf Talman 3
}

\footnotetext{
${ }^{\prime}$ Faculty of Economics and Business Administration, VU University Amsterdam, and Tinbergen Institute;

2Maastricht University;

3 Tilburg University.
} 
Tinbergen Institute is the graduate school and research institute in economics of Erasmus University Rotterdam, the University of Amsterdam and VU University Amsterdam.

More TI discussion papers can be downloaded at http://www.tinbergen.nl

Tinbergen Institute has two locations:

Tinbergen Institute Amsterdam

Gustav Mahlerplein 117

1082 MS Amsterdam

The Netherlands

Tel.: +31(0)205251600

Tinbergen Institute Rotterdam

Burg. Oudlaan 50

3062 PA Rotterdam

The Netherlands

Tel.: +31(0)10 4088900

Fax: $+31(0) 104089031$

Duisenberg school of finance is a collaboration of the Dutch financial sector and universities, with the ambition to support innovative research and offer top quality academic education in core areas of finance.

DSF research papers can be downloaded at: http://www.dsf.nl/

Duisenberg school of finance

Gustav Mahlerplein 117

1082 MS Amsterdam

The Netherlands

Tel.: +31(0)20 5258579 


\title{
The Average Tree Permission Value for Games with a Permission Tree
}

\author{
René van den Brink ${ }^{1}$ P. Jean-Jacques Herings ${ }^{2}$ \\ Gerard van der Laan ${ }^{3}$ Dolf Talman ${ }^{4}$
}

January 3, 2013

${ }^{1}$ J.R. van den Brink, Department of Econometrics and Tinbergen Institute, VU University, De Boelelaan 1105, 1081 HV Amsterdam, The Netherlands. e-mail: jrbrink@feweb.vu.nl

${ }^{2}$ P.J.J. Herings, Department of Economics, Maastricht University, P.O. Box 616, 6200 MD Maastricht, The Netherlands. e-mail: P.Herings@maastrichtuniversity.nl

${ }^{3}$ G. van der Laan, Department of Econometrics and Tinbergen Institute, VU University, De Boelelaan 1105, 1081 HV Amsterdam, The Netherlands. e-mail: glaan@feweb.vu.nl

${ }^{4}$ A.J.J. Talman, CentER, Department of Econometrics and Operations Research, Tilburg University, P.O. Box 90153, 5000 LE Tilburg, The Netherlands, e-mail: talman@tilburguniversity.edu 


\begin{abstract}
In the literature various models of games with restricted cooperation can be found. In those models, instead of allowing for all subsets of the set of players to form, it is assumed that the set of feasible coalitions is a proper subset of the power set of the set of players. In this paper we consider such sets of feasible coalitions that follow from a permission structure on the set of players, in which players need permission to cooperate with other players. We assume the permission structure to be an oriented tree. This means that there is one player at the top of the permission structure and for every other player there is a unique directed path from the top player to this player. We introduce a new solution for these games based on the idea of the Average Tree value for cycle-free communication graph games. We provide two axiomatizations for this new value and compare it with the conjunctive permission value.
\end{abstract}

Keywords: TU game, restricted cooperation, permission structure, Shapley value, Average Tree value, axiomatization.

AMS subject classification: 91A12, 5C20

JEL code: C71 


\section{Introduction}

A cooperative game with transferable utility, or simply a TU game, is a finite set of players and for every coalition of players, a worth representing the total payoff that the coalition can obtain by cooperating. A value is a single-valued solution that assigns to every TU game a payoff vector whose components are the individual payoffs of the players. One of the most applied solutions for cooperative TU games is the Shapley value (Shapley, 1953).

In its classical interpretation, a TU game describes a situation in which the players of every subset of the set of players is able to cooperate to form a feasible coalition and earn its worth. In the literature various restrictions on coalition formation are developed. ${ }^{1}$ For example, in Myerson (1977) a coalition is feasible if it is a connected set in a given communication graph on the set of players. The Myerson value for such so-called graph games is the Shapley value of the corresponding Myerson restricted game in which the worth of any coalition is the sum of the worths of its maximally connected subsets.

On the class of cycle-free graph games, the Average Tree value has been proposed in Herings, van der Laan, and Talman (2008). Each player in a cycle-free graph game can be associated with a particular payoff vector introduced in Demange (2004), called hierarchical outcome. The Average Tree value assigns to every cycle-free graph game the average of its hierarchical outcomes. Both the Myerson value and the Average Tree value are characterized by component efficiency and some kind of fairness. Fairness of the Myerson value states that, after deleting a link between two players, the payoffs of these two players change by the same amount, see Myerson (1977). Component fairness of the Average Tree value states that deleting a link between two players in a cycle-free graph game yields the same average change in payoff over the players in the two resulting components, see Herings et al. (2008).

In van den Brink, Katsev, and van der Laan (2011) games on union closed systems are considered. In such games the collection of feasible coalitions is closed under union, meaning that for any pair of feasible coalitions also their union is feasible. This class of union closed systems contains the class of antimatroids; games on antimatroids have been studied in Algaba, Bilbao, van den Brink, and Jiménez-Losada (2004). An example of an antimatroid is a permission structure, where players need permission from their superiors in a hierarchical structure, given by a directed graph, when they want to cooperate with others. Games with a permission structure are considered in Gilles, Owen, and van den Brink (1992), van den Brink and Gilles (1996), Gilles and Owen (1994), and van den Brink (1997). ${ }^{2}$ In the first two papers the conjunctive approach, in which each

\footnotetext{
${ }^{1}$ For a survey we refer to Bilbao (2000).

${ }^{2}$ Other models of games with a hierarchy on the set of players are, for example, Faigle and Kern (1992) and $\mathrm{Li}$ and $\mathrm{Li}(2011)$.
} 
player needs permission of all its predecessors, is investigated, while in the latter two papers the disjunctive approach is considered, in which a player needs permission of at least one of its predecessors, if it has any. This leads to the conjunctive restricted game and the disjunctive restricted game, in which the worth of a coalition is set equal to its largest conjunctive and its largest disjunctive feasible subcoalition, respectively. The corresponding conjunctive (disjunctive) permission value is then the Shapley value of the induced conjunctive (disjunctive) restricted game. We restrict ourselves to games with an oriented tree as permission structure, i.e., there is a unique top player, having no predecessors, and for every other player there is a unique path from the top player to this player. In this case the conjunctive and disjunctive approaches coincide and we refer simply to the resulting restricted game as the permission game.

In this paper we define and axiomatize a new value for games with an oriented tree as permission structure. Given a digraph we obtain the associated undirected graph by replacing every directed link from one node to another by an undirected link between the two nodes. When the digraph is an oriented tree, the associated undirected graph is cycle-free. To define the new value, we first take the induced permission game and then apply the Average Tree value to this permission game on the associated undirected graph.

We provide two axiomatizations, one with and one without additivity. The first axiomatization uses axioms similar to those that characterize the conjunctive permission value in van den Brink and Gilles (1996), but adding a collusion neutrality axiom in the spirit of Haller (1994) and van den Brink (2012). The second one imposes a fairness property related to the one in Herings et al. (2008) for cycle-free graph games.

The paper is organized as follows. Section 2 is a preliminary section on cooperative TU games, the Average Tree value for cycle-free communication graph games, and games with a permission structure. Section 3 introduces the Average Tree permission value for games with a permission tree structure and provides the first axiomatization. A characterization with a fairness property is given in Section 4. A comparison with the conjunctive permission value is made in Section 5, where we modify the two axiomatizations of the AT permission value to obtain new axiomatizations of the conjunctive permission value. Section 6 contains concluding remarks.

\section{Cooperative games and restricted cooperation}

\subsection{Transferable utility games}

A cooperative game with transferable utility in characteristic function form, or TU game, is a pair $(N, v)$, where $N \subset \mathbb{N}$ is a finite set of $|N|$ players and $v: 2^{N} \rightarrow \mathbb{R}$ is a characteristic function, where $v(\emptyset)=0$. A subset $S \in 2^{N}, S \neq \emptyset$, is called a coalition. For any coalition 
$S, v(S)$ displays the worth of coalition $S$, which the members of coalition $S$ are able to divide among themselves when they decide to cooperate. For given player set $N$, we denote the collection of all TU games on $N$ by $\mathcal{G}^{N}$.

For $T \in 2^{N} \backslash\{\emptyset\}$, the unanimity game $\left(N, u^{T}\right)$ in $\mathcal{G}^{N}$ is given by the characteristic function $u^{T}(S)=1$ if $T \subseteq S$, and $u^{T}(S)=0$ otherwise. For any $(N, v) \in \mathcal{G}^{N}, v$ can be written in a unique way as a linear combination of the characteristic functions $u^{T}$, $T \in 2^{N} \backslash\{\emptyset\}$, as $v=\sum_{T \in 2^{N} \backslash\{\emptyset\}} \Delta_{v}(T) u^{T}$, where the real numbers $\Delta_{v}(T)$ are the Harsanyi dividends, see Harsanyi (1959).

For arbitrary $K \subset \mathbb{N}$, we denote $\mathbb{R}^{K}$ as the $|K|$-dimensional Euclidean space with elements $x \in \mathbb{R}^{K}$ having components $x_{i}, i \in K$. A payoff vector of a game $(N, v) \in \mathcal{G}^{N}$ is a vector $x \in \mathbb{R}^{N}$ giving a payoff $x_{i} \in \mathbb{R}$ to every player $i \in N$. A value for TU games is a single-valued solution $f$ that assigns to every TU game $(N, v) \in \mathcal{G}^{N}$ a payoff vector $f(N, v) \in \mathbb{R}^{N}$. A solution $f$ is efficient if $\sum_{i \in N} f_{i}(N, v)=v(N)$ for every $(N, v) \in \mathcal{G}^{N}$. The best-known solution is the Shapley value, denoted by Sh. This solution is efficient and originally introduced by Shapley (1953) as the solution in which each player receives its average marginal contribution to the coalitions when all orders of entrance (permutations) of the players have equal probability. In terms of Harsanyi dividends the Shapley value is given by $\operatorname{Sh}_{i}(N, v)=\sum_{\left\{T \in 2^{N} \mid i \in T\right\}} \Delta_{v}(T) /|T|, i \in N$, so the Harsanyi dividends $\Delta_{v}(T)$ are distributed uniformly over the players in coalition $T$.

\subsection{TU games with graph structure}

A graph is a pair $(N, L)$ where $N$ is a set of nodes and $L \subseteq\left\{\{i, j\} \in 2^{N} \mid i \neq j\right\}$ is a set of unordered pairs of distinct elements of $N$. In this paper the nodes represent the players in a game $(N, v)$ and so we refer to them as players. The elements of $L$ are called links or edges. For $j \in N$, we denote $N^{L}(j) \subset N$ as the set of neighbors of $j$ in $L$, so $N^{L}(j)=\{h \in N \mid\{j, h\} \in L\}$. The set of all graphs on $N$ is denoted by $\mathcal{L}^{N}$.

For given $S \in 2^{N} \backslash\{\emptyset\}$ and $(N, L) \in \mathcal{L}^{N}$, the graph $(S, L(S))$ with $L(S)=\{\{i, j\} \in$ $L \mid i, j \in S\}$ is the subgraph of $L$ on $S$. Notice that $L(N)=L$. A sequence of $k$ distinct players $\left(i_{1}, \ldots, i_{k}\right)$ is a path in $L(S)$ if $\left\{i_{\ell}, i_{\ell+1}\right\} \in L(S)$ for $\ell=1, \ldots, k-1$. Two players $i, j \in N$ are connected in $(S, L(S))$ if there is a path $\left(i_{1}, \ldots, i_{k}\right)$ in $L(S)$ with $i_{1}=i$ and $i_{k}=j$. A subgraph $(S, L(S))$ is connected, or shortly coalition $S$ is connected, if every two players in $S$ are connected in $(S, L(S))$. A coalition $K \subseteq S$ is a component of $(S, L(S))$ if $K$ is a maximally connected subset of $S$, i.e., $K$ is connected and for every $i \in S \backslash K$ the set $K \cup\{i\}$ is not connected. The set of components of $(S, L(S))$ is denoted by $\Sigma^{L}(S)$, with $\Sigma^{L}=\Sigma^{L}(N)$. The graph $(N, L)$ is cycle-free if for every two different players $i$ and $j$ either $i$ and $j$ are not connected or there is precisely one path in $L$ connecting $i$ and $j$. When $(N, L)$ is connected and cycle-free, then $N$ is the unique component of $(N, L)$ and 
$(N, L)$ has precisely $|N|-1$ links. Following Béal, Rémila and Solal (2010) (see also Béal, Lardon, Rémila and Solal (2012) for multi-choice forest games), we call $N$ as well as each of the two components in $(N, L \backslash\{\{i, j\}\})$ a cone when $(N, L)$ is connected and cycle-free. Therefore, a connected cycle-free graph $(N, L)$ has $2(|N|-1)+1$ cones.

A $T U$ game with graph structure, shortly graph game, is a triple $(N, v, L)$ with $(N, v) \in \mathcal{G}^{N}$ and $(N, L) \in \mathcal{L}^{N}$. We denote the collection of all TU games with graph structure and player set $N$ by $\mathcal{G}_{\mathcal{L}}^{N}$ and the class of all cycle-free graph games on $N$ by $\mathcal{G}_{\mathcal{F}}^{N}$. A solution $f$ on a subclass $\mathcal{G}$ of $\mathcal{G}_{\mathcal{L}}^{N}$ assigns a unique payoff vector $f(N, v, L) \in \mathbb{R}^{N}$ to every $(N, v, L) \in \mathcal{G}$.

For a graph game $(N, v, L)$, Myerson (1977) introduced the Myerson restricted game $\left(N, v^{L}\right) \in \mathcal{G}^{N}$, defined by $v^{L}(S)=\sum_{T \in \Sigma^{L}(S)} v(T)$ for every $S \in 2^{N}$. The Myerson value, denoted by $\mathrm{My}$, is defined as $\operatorname{My}(N, v, L)=\operatorname{Sh}\left(N, v^{L}\right)$, for every $(N, v, L) \in \mathcal{G}_{\mathcal{L}}^{N}$. The Myerson value is characterized by component efficiency and fairness, where a solution $f$ is component efficient if for any $(N, v, L) \in \mathcal{G}_{\mathcal{L}}^{N}$ it holds that $\sum_{i \in K} f_{i}(N, v, L)=v(K)$ for every $K \in \Sigma^{L}$ and solution $f$ satisfies fairness if for any $(N, v, L) \in \mathcal{G}_{\mathcal{L}}^{N}$ and any link $\{i, j\} \in L$ it holds that $f_{i}(N, v, L)-f_{i}(N, v, L \backslash\{\{i, j\}\})=f_{j}(N, v, L)-f_{j}(N, v, L \backslash\{\{i, j\}\})$.

On the class $\mathcal{G}_{\mathcal{F}}^{N}$ of cycle-free graph games, Herings et al. (2008) introduce the Average Tree value, denoted by AT. When the graph is connected, the AT value assigns to each graph game $(N, v, L) \in \mathcal{G}_{\mathcal{F}}^{N}$ the average of $|N|$ payoff vectors. Each of these payoff vectors is associated with precisely one of the players, the so-called hierarchical outcome for that player as introduced by Demange (2004). To define the hierarchical outcome for a particular player $i \in N$, for each $j \in N$ let $C_{i}^{L}(j)$ be defined as

$$
C_{i}^{L}(j)=\{h \in N \mid \text { the path in } L \text { from } h \text { to } i \text { contains } j\} .
$$

Notice that $C_{i}^{L}(i)=N$ and, for $j \neq i, C_{i}^{L}(j)$ is the cone containing $j$ that results from deleting the first link of the unique path in $L$ from $j$ to $i$. The hierarchical outcome associated to player $i$ is the vector $t^{i}(N, v, L) \in \mathbb{R}^{N}$ defined as

$$
t_{j}^{i}(N, v, L)=v\left(C_{i}^{L}(j)\right)-\sum_{h \in C_{i}^{L}(j) \cap N^{L}(j)} v\left(C_{i}^{L}(h)\right), \quad j \in N .
$$

The payoff to player $j$ in this vector is equal to the worth of the cone $C_{i}^{L}(j)$ minus the worths of the cones $C_{i}^{L}(h)$ for the neighbors $h$ of $j$ in $C_{i}^{L}(j)$. Since $t_{i}^{i}(N, v, L)=v(N)-$ $\sum_{h \in N^{L}(i)} v\left(C_{i}^{L}(h)\right)$, the hierarchical outcome $t^{i}(N, v, L)$ is efficient.

On the class of connected cycle-free graph games, the AT value is then defined as

$$
\operatorname{AT}(N, v, L)=\frac{1}{|N|} \sum_{i \in N} t^{i}(N, v, L) .
$$

If $(N, L)$ is connected, the AT value depends only on the worths of the $2(|N|-1)+1$ cones. When $(N, L)$ is not connected, the AT value is applied to each of the components 
in $\Sigma^{L}$, i.e., on each component $K$ the AT value is the average of $|K|$ hierarchical outcomes of length $K$ associated to each of the players in $K$. This construction defines the AT value on the class of cycle-free graph games $\mathcal{G}_{\mathcal{F}}^{N}$.

On the class $\mathcal{G}_{\mathcal{F}}^{N}$, the AT value is characterized by component efficiency and component fairness. For $(N, v, L) \in \mathcal{G}_{\mathcal{F}}^{N}$, take $K \in \Sigma^{L}$ and link $\{i, j\} \in L(K)$. Then $K$ consists of two components in the graph $(N, L \backslash\{\{i, j\}\})$, obtained from $(N, L)$ by deleting the link $\{i, j\}$. Let $K_{h}^{i j}, h=i, j$, denote the component of $K$ that contains player $h$ after deleting the link $\{i, j\} .{ }^{3}$ Component fairness requires that, when deleting link $\{i, j\}$ in $L(K)$, the resulting average change in payoff to the players in $K_{i}^{i j}$ is equal to the average change in payoff to the players in $K_{j}^{i j}$.

\section{Axiom 2.1 Component Fairness}

A solution $f$ on the class $\mathcal{G}_{\mathcal{F}}^{N}$ of cycle-free graph games satisfies component fairness if, for every $(N, v, L) \in \mathcal{G}_{\mathcal{F}}^{N}$ and for any link $\{i, j\} \in L$, it holds that

$$
\frac{\sum_{h \in K_{i}^{i j}}\left[f_{h}(N, v, L)-f_{h}(N, v, L \backslash\{\{i, j\}\})\right]}{\left|K_{i}^{i j}\right|}=\frac{\sum_{h \in K_{j}^{i j}}\left[f_{h}(N, v, L)-f_{h}(N, v, L \backslash\{\{i, j\}\})\right]}{\left|K_{j}^{i j}\right|} .
$$

\section{Theorem 2.2 (Herings, van der Laan, and Talman, 2008)}

On the class $\mathcal{G}_{\mathcal{F}}^{N}$ of cycle-free graph games, the AT value is the unique solution that satisfies component efficiency and component fairness.

Both the Myerson value and the AT value satisfy efficiency on the class of connected cycle-free graph games.

\subsection{TU games with permission structure}

A permission structure on the set of players of a TU game describes a situation where some players need permission from other players to cooperate within a coalition. A permission structure is assumed to be described by a directed graph, shortly digraph, $(N, D)$ with the finite set of players of the game $N$ as the set of nodes and with set of $\operatorname{arcs} D \subseteq\{(i, j) \in N \times$ $N \mid i \neq j\}$ a collection of ordered pairs of players in $N$. For a digraph $(N, D)$, the undirected graph $\left(N, L_{D}\right)$ on $N$ associated to $D$ is defined by $L_{D}=\left\{\{i, j\} \in 2^{N} \backslash\{\emptyset\} \mid(i, j) \in D\right\}$. The digraph $(N, D)$ is connected if $\left(N, L_{D}\right)$ is connected, and a coalition $K \in 2^{N} \backslash\{\emptyset\}$ is a component of $(N, D)$ if it is a component of $\left(N, L_{D}\right)$.

For a given digraph $(N, D)$, node $i$ is a predecessor of $j$ and $j$ is a successor of $i$ if $(i, j) \in D$. A directed path in $(N, D)$ from $i$ to $j$ is a sequence of distinct nodes $\left(i_{1}, \ldots, i_{m}\right)$ such that $i_{1}=i, i_{m}=j$, and $\left(i_{k}, i_{k+1}\right) \in D$ for $k=1, \ldots, m-1$. If there is a directed path

\footnotetext{
${ }^{3}$ When $(N, L)$ is connected, then $K_{h}^{i j}, h=i, j$, are the two cones in $L$ that result from deleting $\{i, j\}$.
} 
in $(N, D)$ from node $i$ to a different node $j$, then $i$ is a superior of $j$ and $j$ is a subordinate of $i$. A directed path from $i$ to $j$ is a cycle in $(N, D)$ if $(j, i) \in D$. The digraph $(N, D)$ is acyclic if it does not admit cycles. An acyclic digraph on a finite set has at least one top node, being a node that has no predecessors. A digraph $(N, D)$ is an oriented tree if it has only one top node and from the top node to any other node there is precisely one directed path in the digraph. The collection of all oriented trees on $N$ is denoted by $\mathcal{D}_{\mathcal{T}}^{N}$. The associated undirected graph $\left(N, L_{D}\right)$ of an oriented tree $(N, D) \in \mathcal{D}_{\mathcal{T}}^{N}$ is both connected and cycle-free.

For an oriented tree $(N, D) \in \mathcal{D}_{\mathcal{T}}^{N}$ every node $j \in N$, except the top node, has a unique predecessor, denoted by $p_{D}(j)$. For $i \in N, S_{D}(i)=\{j \in N \mid(i, j) \in D\}$ denotes the set of successors, $\widehat{S}_{D}(i)$ denotes the set of subordinates, and $\widehat{P}_{D}(i)$ denotes the set of superiors of node $i$. Notice that in an oriented tree the top node is a superior of any other node and that any other node is a subordinate of the top node. Finally, for $T \in 2^{N}$, we denote $\widehat{S}_{D}(T)=\cup_{i \in T} \widehat{S}_{D}(i)$ and $\widehat{P}_{D}(T)=\cup_{i \in T} \widehat{P}_{D}(i)$. Let $i_{0}$ be the top node of $(N, D) \in \mathcal{D}_{\mathcal{T}}^{N}$, so $\widehat{S}_{D}\left(i_{0}\right)=N \backslash\left\{i_{0}\right\}$.

A TU game with permission structure is a triple $(N, v, D)$ with player set $N$, TU game $(N, v) \in \mathcal{G}^{N}$, and digraph $(N, D)$ on the set of players. A solution $f$ on a class of games with permission structure assigns a unique payoff vector $f(N, v, D) \in \mathbb{R}^{N}$ to every $(N, v, D)$ in the class. In a game with permission structure it is assumed that players need permission of their predecessors to cooperate with other players. In the conjunctive approach as introduced in Gilles et al. (1992) and van den Brink and Gilles (1996) it is assumed that a player needs permission from all its predecessors, while in the disjunctive approach as considered in Gilles and Owen (1994) and van den Brink (1997) it is assumed that a player needs permission of at least one of its predecessors if it has any.

In this paper we consider the class of games with permission tree structure, shortly permission tree games and denote this collection of games by $\mathcal{G}_{\mathcal{T}}^{N}$. On this class the conjunctive and disjunctive approaches coincide and a coalition $S \in 2^{N}$ is feasible if for every player $j \in S$ all its predecessors are members of $S$. It follows that all its superiors, including the top node player, are members of $S$. The smallest feasible coalition containing $S$ is equal to $F(S)=S \cup \widehat{P}_{D}(S)$. The set of feasible coalitions is given by

$$
\Omega_{D}=\left\{S \in 2^{N} \mid \forall i \in S \backslash\left\{i_{0}\right\}, p_{D}(i) \in S\right\}=\left\{S \in 2^{N} \mid F(S)=S\right\} .
$$

As shown by Algaba et al. (2004), the collection $\Omega_{D}$ is an antimatroid and is therefore union closed, ${ }^{4}$ i.e., for every two sets $S, T \in \Omega_{D}$ it holds that $S \cup T \in \Omega_{D}$. Therefore, for any $S \in 2^{N}$, the largest feasible subset of $S$ is uniquely defined and is equal to

\footnotetext{
${ }^{4}$ A collection of feasible coalitions $\mathcal{A} \subseteq 2^{N}$ is an antimatroid if, besides being union closed, it contains the empty set and it satisfies accessibility meaning that $S \in \mathcal{A}$ implies that there is a player $i \in S$ such that $S \backslash\{i\} \in \mathcal{A}$, see Dilworth (1940) and Edelman and Jamison (1985).
} 
$\sigma_{D}(S)=\cup_{\left\{T \in \Omega_{D} \mid T \subseteq S\right\}} T$. The induced permission restricted game of a permission tree game $(N, v, D) \in \mathcal{G}_{\mathcal{T}}^{N}$ is the game $\left(N, v_{D}\right) \in \mathcal{G}^{N}$ given by

$$
v_{D}(S)=v\left(\sigma_{D}(S)\right), \quad S \in 2^{N}
$$

i.e., the permission restricted game assigns to each coalition $S \in 2^{N}$ the worth of the largest feasible subset of $S$ in the game $(N, v)$.

The conjunctive permission value $\varphi$ is the solution that assigns to every game $(N, v, D) \in \mathcal{G}_{\mathcal{T}}^{N}$ the Shapley value of the permission restricted game, $\varphi(N, v, D)=\operatorname{Sh}\left(N, v_{D}\right)$.

\section{The Average Tree permission value}

In this section, we introduce a new value on the class of permission tree games and characterize it by a set of six independent axioms. The new value can be computed by applying the AT value to the cycle-free graph game $\left(N, v_{D}, L_{D}\right)$ which is associated with the permission tree game $(N, v, D) \in \mathcal{G}_{\mathcal{T}}^{N}$.

\section{Definition 3.1 AT permission value}

On the class $\mathcal{G}_{\mathcal{T}}^{N}$ of permission tree games, the Average Tree (AT) permission value is the function $\psi$ given by

$$
\psi(N, v, D)=\operatorname{AT}\left(N, v_{D}, L_{D}\right),(N, v, D) \in \mathcal{G}_{\mathcal{T}}^{N}
$$

To obtain the AT permission value of a permission tree game $(N, v, D)$, first the permission restricted game $\left(N, v_{D}\right)$ is taken and then the Average Tree value is applied with $\left(N, L_{D}\right)$ as the underlying graph.

We give a first characterization of the AT permission value by means of six independent axioms. The first three axioms are also used in van den Brink and Gilles (1996) to characterize the conjunctive permission value. Efficiency states that the total sum of payoffs equals the worth of the grand coalition.

\section{Axiom 3.2 Efficiency}

For every $(N, v, D) \in \mathcal{G}_{\mathcal{T}}^{N}$ it holds that $\sum_{i \in N} f_{i}(N, v, D)=v(N)$.

Linearity is a straightforward generalization of the linearity axiom for TU games.

\section{Axiom 3.3 Linearity}

For every pair $(N, v, D),(N, w, D) \in \mathcal{G}_{\mathcal{T}}^{N}$ and real numbers $\alpha$ and $\beta$ it holds that $f(N, \alpha v+$ $\beta w, D)=\alpha f(N, v, D)+\beta f(N, w, D)$. 
Player $i \in N$ is a null player in $(N, v) \in \mathcal{G}^{N}$ if for all $T \subseteq N \backslash\{i\}$ it holds that $v(T \cup\{i\})-v(T)=0$. Player $i \in N$ is an inessential player in the permission tree game $(N, v, D) \in \mathcal{G}_{\mathcal{T}}^{N}$ if both $i$ and all its subordinates in $(N, D)$ are null players in $(N, v)$. The inessential player property states that inessential players earn zero payoff. ${ }^{5}$

\section{Axiom 3.4 Inessential player property}

For every $(N, v, D) \in \mathcal{G}_{\mathcal{T}}^{N}$ it holds that if player $i \in N$ is an inessential player in $(N, v, D)$, then $f_{i}(N, v, D)=0$.

For $T \in 2^{N}$, we call $\operatorname{Int}_{D}(T)=\left\{i \in T \mid N^{L_{D}}(i) \subset T\right\}$ the interior of coalition $T$ in $(N, D)$. This set consists of all players in $T$ whose neighbors all belong to $T$. Considering the unanimity game of $T$, the players in the interior of $T$ have no links going outside of $T$, and thus they can only directly communicate with players within $T$. The next axiom is a weak symmetry axiom and states that when $(N, v)$ is the unanimity game of some coalition $T$ then all players in the interior of $T$ get the same payoff.

\section{Axiom 3.5 Interior unanimity symmetry}

For every $T \in 2^{N} \backslash\{\emptyset\},\left(N, u^{T}, D\right) \in \mathcal{G}_{\mathcal{T}}^{N}$, and $i, j \in \operatorname{Int}_{D}(T)$ it holds that $f_{i}\left(N, u^{T}, D\right)=$ $f_{j}\left(N, u^{T}, D\right)$.

Interior unanimity symmetry is related to the communication ability property for graph games introduced by Borm, Owen, and Tijs (1992) to characterize the Myerson value. For connected graph games this latter axiom states that if all players are symmetric in the Myerson restricted game, then they all get the same payoff. In van den Brink, van der Laan, and Pruzhansky (2011) this is weakened by requiring this only for the unanimity game on the grand coalition.

The next axiom reflects predecessor necessity and states that in case of a unanimity game with respect to $T$, the payoff distribution does not change if the predecessor of a player in $T$ is added to the unanimity game.

\section{Axiom 3.6 Predecessor necessity}

For every $T \in 2^{N} \backslash\{\emptyset\},\left(N, u^{T}, D\right) \in \mathcal{G}_{\mathcal{T}}^{N}$, and $j \in T$ it holds that $f\left(N, u^{T \cup\left\{p_{D}(j)\right\}}, D\right)=$ $f\left(N, u^{T}, D\right)$.

Interior unanimity symmetry and predecessor necessity are also satisfied by the conjunctive permission value. The next axiom is not satisfied by the conjunctive permission value. For TU games, Haller (1994) considers collusion neutrality properties, one of them stating that when two players act together in the sense that either both players are together

\footnotetext{
${ }^{5}$ It weakens the null player property, which states that a null player earns zero payoff.
} 
in a coalition or both stay out of a coalition, then the sum of the payoffs of the two players does not change. For a similar collusion neutrality property, van den Brink (2012) shows that there is no solution for TU games that satisfies efficiency, collusion neutrality, and the null player property, while on the class of communication graph games, all hierarchical outcomes and their convex combinations, and thus also the AT value, do satisfy these three properties when only collusion is allowed among neighbors. Here we restrict the axiom to any two players that are neighbors in the permission structure. For a game $(N, v) \in \mathcal{G}^{N}$ and two players $i, j \in N$, the game in which players $i$ and $j$ act together is defined as the game $\left(N, v^{i j}\right) \in \mathcal{G}^{N}$ given by $v^{i j}(T)=v(T \backslash\{i, j\})$ if $\{i, j\} \nsubseteq T$, and $v^{i j}(T)=v(T)$ otherwise.

\section{Axiom 3.7 Collusion neutrality}

For every $(N, v, D) \in \mathcal{G}_{\mathcal{T}}^{N}$ and $i, j \in N$ with $j \in N^{L_{D}}(i)$ it holds that $f_{i}\left(N, v^{i j}, D\right)+$ $f_{j}\left(N, v^{i j}, D\right)=f_{i}(N, v, D)+f_{j}(N, v, D)$.

The AT permission value is characterized by Axioms 3.2-3.7.

Theorem 3.8 On the class $\mathcal{G}_{\mathcal{T}}^{N}$ of permission tree games, the AT permission value is the unique solution that satisfies efficiency, linearity, the inessential player property, interior unanimity symmetry, predecessor necessity, and collusion neutrality.

Proof. For notational convenience, in the proof we denote the cone $C_{i}^{L_{D}}(j)$ of player $j$ in the associated undirected graph $\left(N, L_{D}\right)$ of $(N, D)$ with respect to $i$ by $C_{i}^{D}(j)$ and the set of neighbors $N^{L_{D}}(j)$ of player $j$ by $N^{D}(j)$.

We first verify that the AT permission value satisfies all six axioms. Take any $(N, v, D) \in \mathcal{G}_{\mathcal{T}}^{N}$.

1. Since $(N, D) \in \mathcal{D}_{\mathcal{T}}^{N}$, the associated undirected graph $\left(N, L_{D}\right)$ is connected and so every vector $t^{i}\left(N, v_{D}, L_{D}\right), i \in N$, is efficient with respect to $v_{D}$, thus $\sum_{k \in N} t_{k}^{i}\left(N, v_{D}, L_{D}\right)=$ $v_{D}(N)$. Also, since $(N, D) \in \mathcal{D}_{\mathcal{T}}^{N}$, it holds that $N \in \Omega_{D}$, and thus $v_{D}(N)=v(N)$. It follows that $\psi(N, v, D)$ is efficient.

2. Consider $(N, v)$ and $(N, w)$ in $\mathcal{G}^{N}$, real numbers $\alpha, \beta$, and define $z=\alpha v+\beta w$. Since $z_{D}(S)=z\left(\sigma_{D}(S)\right)=\alpha v\left(\sigma_{D}(S)\right)+\beta w\left(\sigma_{D}(S)\right)=\alpha v_{D}(S)+\beta w_{D}(S)$ for every $S \in 2^{N}$, and $t^{i}$ is linear in its second argument, for every $i \in N$, it follows that $t^{i}\left(N, z_{D}, L_{D}\right)=$ $t^{i}\left(N, \alpha v_{D}+\beta w_{D}, L_{D}\right)=\alpha t^{i}\left(N, v_{D}, L_{D}\right)+\beta t^{i}\left(N, w_{D}, L_{D}\right)$. Since the AT permission value is the average over the vectors $t^{i}$, it follows that $\psi$ is linear.

3 . Let $j$ be an inessential player in $(N, v, D)$. We distinguish two cases.

Case 1. When $j$ is the unique top node in $D$, then all players are null players and $v(T)=0$ for all $T \in 2^{N}$. It follows that $v_{D}(T)=0$ for every $T \in 2^{N}$ and thus $t_{j}^{i}\left(N, v_{D}, L_{D}\right)=0$ for all $i \in N$. Taking the average over all $i \in N$ yields $\psi_{j}\left(N, v_{D}, L_{D}\right)=0$. 
Case 2. Next we consider the case that $j$ is not the top node of $(N, D)$. Take an arbitrary player $i \in N$ and consider the vector $t^{i}\left(N, v_{D}, L_{D}\right)$. We show that $t_{j}^{i}\left(N, v_{D}, L_{D}\right)=0$.

First, when $i$ is a subordinate of $j$, then $C_{i}^{D}(j) \cap N^{D}(j)$ contains the unique predecessor of $j$, say $h$, while all other players in this set are successors of $j$. So,

$$
t_{j}^{i}\left(N, v_{D}, L_{D}\right)=v_{D}\left(C_{i}^{D}(j)\right)-v_{D}\left(C_{i}^{D}(h)\right)-\sum_{k \in C_{i}^{D}(j) \cap S_{D}(j)} v_{D}\left(C_{i}^{D}(k)\right) .
$$

For every $k \in C_{i}^{D}(j) \cap S_{D}(j)$, the set $C_{i}^{D}(k)$ is a set of subordinates of $j$ and so $\sigma_{D}\left(C_{i}^{D}(k)\right)=$ $\emptyset$. Further, since $D$ is an oriented tree, both $C_{i}^{D}(j)$ and $C_{i}^{D}(h)$ are feasible in $(N, v, D)$. Hence,

$$
t_{j}^{i}\left(N, v_{D}, L_{D}\right)=v\left(C_{i}^{D}(j)\right)-v\left(C_{i}^{D}(h)\right) .
$$

Since $C_{i}^{D}(j) \backslash C_{i}^{D}(h) \subset \widehat{S}_{D}(j) \cup\{j\}$ and $j$ is inessential, it follows that $v\left(C_{i}^{D}(j)\right)=v\left(C_{i}^{D}(h)\right)$, and thus $t_{j}^{i}\left(N, v_{D}, L_{D}\right)=0$.

Second, when $i$ is not a subordinate of $j$ and $i \neq j$, then $C_{i}^{D}(j)=\widehat{S}_{D}(j) \cup\{j\}$ and the neighbors of $j$ within this set are his successors. So $C_{i}^{D}(j) \cap N_{i}^{D}(j)=S_{D}(j)$, and thus

$$
t_{j}^{i}\left(N, v_{D}, L_{D}\right)=v_{D}\left(C_{i}^{D}(j)\right)-\sum_{h \in S_{D}(j)} v_{D}\left(C_{i}^{D}(h)\right)=0
$$

where the last equality follows from the fact that $j$ is not the top node in $(N, D)$, and therefore $\sigma_{D}\left(C_{i}^{D}(k)\right)=\emptyset$ for every $k \in S_{D}(j) \cup\{j\}{ }^{6}$

Third, we consider $i=j$. Since $j$ is not the top node in $D, j$ has precisely one predecessor, say player $k$. From $C_{j}^{D}(k)=N \backslash\left(\widehat{S}_{D}(j) \cup\{j\}\right)$ it follows that

$$
\begin{aligned}
t_{j}^{j}\left(N, v_{D}, L_{D}\right) & =v_{D}(N)-v_{D}\left(N \backslash\left(\widehat{S}_{D}(j) \cup\{j\}\right)\right)-\sum_{h \in S_{D}(j)} v_{D}\left(C_{j}^{D}(h)\right) \\
& =v(N)-v\left(N \backslash\left(\widehat{S}_{D}(j) \cup\{j\}\right)\right)-\sum_{h \in S_{D}(j)} v(\emptyset)=0,
\end{aligned}
$$

where the last equality follows from the fact that $j$ is inessential, so $v\left(N \backslash\left(\widehat{S}_{D}(j) \cup\{j\}\right)\right)=$ $v(N)$. So, $\psi$ satisfies the inessential player property.

4. Let $T \in 2^{N}$ and $j \in T$ be such that $N^{D}(j) \subset T$. Take an arbitrary player $i \neq j$ and consider the vector $t^{i}\left(N, v_{D}, L_{D}\right)$. It holds that

$$
\begin{aligned}
t_{j}^{i}\left(N, u_{D}^{T}, L_{D}\right) & =u_{D}^{T}\left(C_{i}^{D}(j)\right)-\sum_{h \in C_{i}^{D}(j) \cap N^{D}(j)} u_{D}^{T}\left(C_{i}^{D}(h)\right) \\
& =u^{T}\left(\sigma_{D}\left(C_{i}^{D}(j)\right)\right)-\sum_{h \in C_{i}^{D}(j) \cap N^{D}(j)} u^{T}\left(\sigma_{D}\left(C_{i}^{D}(h)\right)\right) .
\end{aligned}
$$

Consider some $h \in C_{i}^{D}(j) \cap N^{D}(j)$. Since $\sigma_{D}\left(C_{i}^{D}(h)\right) \subseteq C_{i}^{D}(h)$ and $j \notin C_{i}^{D}(h)$ it holds that $j \in T \backslash \sigma_{D}\left(C_{i}^{D}(h)\right)$, so $u^{T}\left(\sigma_{D}\left(C_{i}^{D}(h)\right)\right)=0$. Since $N^{D}(j) \subset T$ and $i \neq j$, there exists

\footnotetext{
${ }^{6}$ Note that the last equality also follows because all players in $\widehat{S}_{D}(j) \cup\{j\}$ are null players in $\left(N, v_{D}\right)$.
} 
a player $k \in N^{D}(j) \cap T$ that does not belong to $C_{i}^{D}(j)$, so also not to $\sigma_{D}\left(C_{i}^{D}(j)\right)$. Since $k \in T \backslash \sigma_{D}\left(C_{i}^{D}(j)\right)$, we have that $u^{T}\left(\sigma_{D}\left(C_{i}^{D}(j)\right)\right)=0$.

When $i=j$, then

$$
t_{j}^{j}\left(N, u_{D}^{T}, L_{D}\right)=u_{D}^{T}(N)-\sum_{h \in N^{D}(j)} u_{D}^{T}\left(C_{j}^{D}(h)\right)=u^{T}(N)-\sum_{h \in N^{D}(j)} u^{T}\left(\sigma_{D}\left(C_{j}^{D}(h)\right)\right)=1,
$$

where the second equality follows from $N \in \Omega_{D}$ and the last equality follows because for every $h \in N^{D}(j)$ it holds that $\sigma_{D}\left(C_{j}^{D}(h)\right)$ does not contain $j \in T$. Taking the average over all $i \in N$, it follows that $\psi_{j}\left(N, u^{T}, D\right)=\frac{1}{|N|}$, independent of the choice of $j \in T$ such that $N^{D}(j) \subset T$. So, $\psi$ satisfies interior unanimity symmetry.

5. Take $T \in 2^{N}$ and $j \in T$. We show that $u_{D}^{T}=u_{D}^{T \cup\left\{p_{D}(j)\right\}}$. If $T \nsubseteq S$, then both $T \nsubseteq \sigma_{D}(S)$ and $T \cup\left\{p_{D}(j)\right\} \nsubseteq \sigma_{D}(S)$, which implies $u_{D}^{T \cup\left\{p_{D}(j)\right\}}(S)=u_{D}^{T}(S)=0$. If $T \subseteq S$ and $T \nsubseteq \sigma_{D}(S)$, then also $T \cup\left\{p_{D}(j)\right\} \nsubseteq \sigma_{D}(S)$, which implies $u_{D}^{T \cup\left\{p_{D}(j)\right\}}(S)=u_{D}^{T}(S)=0$. Finally, consider the case $T \subseteq S$ and $T \subseteq \sigma_{D}(S)$. It holds that $u_{D}^{T}(S)=u^{T}\left(\sigma_{D}(S)\right)=1$. Since $T \subseteq \sigma_{D}(S)$ and $\sigma_{D}(S) \in \Omega_{D}$, it holds that $F(T) \subseteq \sigma_{D}(S)$, so in particular $p_{D}(j) \in$ $\sigma_{D}(S)$. We have that $u_{D}^{T \cup\left\{p_{D}(j)\right\}}(S)=u^{T \cup\left\{p_{D}(j)\right\}}\left(\sigma_{D}(S)\right)=1$. So, $\psi$ satisfies predecessor necessity.

6. Let $j \in N^{D}(i)$ and without loss of generality assume that $i=p_{D}(j)$. Let $S \in 2^{N}$ be a set that contains both $i$ and $j$. It holds that $j \in \sigma_{D}(S)$ if and only if $i \in \sigma_{D}(S)$. Now, for some $k \in N$, consider $t^{k}\left(N, v_{D}, L_{D}\right)$.

First, suppose that $j \in C_{k}^{D}(i)$. We define $O_{i}=\left(C_{k}^{D}(i) \cap N^{D}(i)\right) \backslash\{j\}$ and $O_{j}=$ $C_{k}^{D}(j) \cap N^{D}(j)$. Notice that $i \notin O_{j}$ because $j \in C_{k}^{D}(i)$. It holds that

$$
\begin{aligned}
& t_{i}^{k}\left(N, v_{D}, L_{D}\right)=v_{D}\left(C_{k}^{D}(i)\right)-v_{D}\left(C_{k}^{D}(j)\right)-\sum_{h \in O_{i}} v_{D}\left(C_{k}^{D}(h)\right), \\
& t_{j}^{k}\left(N, v_{D}, L_{D}\right)=v_{D}\left(C_{k}^{D}(j)\right)-\sum_{h \in O_{j}} v_{D}\left(C_{k}^{D}(h)\right) .
\end{aligned}
$$

We obtain that

$$
\begin{aligned}
t_{i}^{k}\left(N, v_{D}, L_{D}\right)+t_{j}^{k}\left(N, v_{D}, L_{D}\right) & =v_{D}\left(C_{k}^{D}(i)\right)-\sum_{h \in O_{i} \cup O_{j}} v_{D}\left(C_{k}^{D}(h)\right) \\
& =v\left(\sigma_{D}\left(C_{k}^{D}(i)\right)\right)-\sum_{h \in O_{i} \cup O_{j}} v\left(\sigma_{D}\left(C_{k}^{D}(h)\right)\right) \\
& =v^{i j}\left(\sigma_{D}\left(C_{k}^{D}(i)\right)\right)-\sum_{h \in O_{i} \cup O_{j}} v^{i j}\left(\sigma_{D}\left(C_{k}^{D}(h)\right)\right) \\
& =t_{i}^{k}\left(N,\left(v^{i j}\right)_{D}, L_{D}\right)+t_{j}^{k}\left(N,\left(v^{i j}\right)_{D}, L_{D}\right),
\end{aligned}
$$

where the third equality follows because both $i$ and $j$ are in $C_{k}^{D}(i)$ and thus either both are in $\sigma_{D}\left(C_{k}^{D}(i)\right)$ or both are not, and $i$ and $j$ are both not in $C_{k}^{D}(h)$ for every $h \in O_{i} \cup O_{j}$ and so also not in $\sigma_{D}\left(C_{k}^{D}(h)\right)$. Similar, the same equality holds when $i \in C_{k}^{D}(j)$. By taking the average over all $k \in N$, it follows that $\psi$ satisfies collusion neutrality.

Next we prove that the six axioms determine a unique solution $f$. First, for the unanimity game $\left(N, u^{N}, D\right)$ it holds by efficiency and interior unanimity symmetry that $f_{i}\left(N, u^{N}, D\right)=$ 
$1 /|N|, i \in N$. Next we determine by induction the payoffs of the unanimity games of all feasible sets.

Take any $t, 1 \leq t<|N|$, and assume that $f\left(N, u^{T}, D\right)$ is uniquely determined for all $T \in \Omega_{D}$ with $|T|>t$. Take any $T \in \Omega_{D}$ with $|T|=t$. Since $T$ is feasible, for every $i \notin T$ it holds that also all subordinates of $i$ are not in $T$ and so $i$ is inessential in $\left(N, u^{T}, D\right)$. Thus, for any $i \notin T, f_{i}\left(N, u^{T}, D\right)=0$ by the inessential player property. To determine the payoffs of the players in $T$, for a player $i \in T$ such that $S_{D}(i) \backslash T \neq \emptyset$, take a player $j \in S_{D}(i) \backslash T$. Since $T$ is feasible also $T^{\prime}=T \cup\{j\}$ is feasible and $\left|T^{\prime}\right|=t+1$. Since $j \notin T$, $f_{j}\left(N, u^{T}, D\right)=0$. Applying collusion neutrality to $v=u^{T}$ and observing that $v^{i j}=u^{T^{\prime}}$ it follows that

$$
\begin{aligned}
f_{i}\left(N, u^{T}, D\right) & =f_{i}\left(N, u^{T}, D\right)+f_{j}\left(N, u^{T}, D\right) \\
& =f_{i}\left(N,\left(u^{T}\right)^{i j}, D\right)+f_{j}\left(N,\left(u^{T}\right)^{i j}, D\right) \\
& =f_{i}\left(N, u^{T^{\prime}}, D\right)+f_{j}\left(N, u^{T^{\prime}}, D\right) .
\end{aligned}
$$

By the induction hypothesis, $f_{i}\left(N, u^{T^{\prime}}, D\right)$ and $f_{j}\left(N, u^{T^{\prime}}, D\right)$ are uniquely determined, and therefore $f_{i}\left(N, u^{T}, D\right)$ is uniquely determined. So, we are left to determine the payoffs of the players in the set $\widehat{T}=\left\{h \in T \mid S_{D}(h) \backslash T=\emptyset\right\}$. For every $i \in \widehat{T}$ it holds that $N^{D}(i) \subset T$, because $T$ is feasible and $S_{D}(i) \backslash T=\emptyset$. From interior unanimity symmetry it follows that all players in $\widehat{T}$ have equal payoff. These payoffs then follow from efficiency. By induction it is shown that $f\left(N, u^{T}, D\right)$ is uniquely determined for every feasible $T \in \Omega_{D}$.

Take any $T \notin \Omega_{D}$. Predecessor necessity implies $f\left(N, u^{T}, D\right)=f\left(N, u^{T \cup\left\{p_{D}(j)\right\}}, D\right)$ for every $j \in T$. Adding subsequently all players in $\widehat{P}_{D}(T) \backslash T$, we obtain that $f\left(N, u^{T}, D\right)=$ $f\left(N, u^{F(T)}, D\right)$ and so the payoffs for every unanimity game are uniquely determined.

Finally, $f(N, v, D)$ is uniquely determined by linearity for every $(N, v, D) \in \mathcal{G}_{\mathcal{T}}^{N}$.

Note that collusion between two neighbors in the restricted game is not the same as taking the restricted game after two neighbors colluded. Consider, for example the game with permission structure $(N, v, D) \in \mathcal{G}_{\mathcal{T}}^{N}$ with $N=\{1,2,3,4\}, v=u^{\{1,3\}}$, and $D=\{(1,2),(2,3),(2,4)\}$. Taking $S=\{1,2,3\}$ we see that $\left(v_{D}\right)^{24}(S)=v_{D}(\{1,3\})=$ $v(\{1\})=0$, while $\left(v^{24}\right)_{D}(S)=v^{24}(\{1,2,3\})=v(\{1,3\})$. If $\left(v^{i j}\right)_{D}$ would always be equal to $\left(v_{D}\right)^{i j}$, then collusion neutrality would follow immediately from van den Brink (2012), who shows that when two neighbors in an undirected communication graph collude then the sum of their payoffs does not change in every hierarchical outcome, and thus any of their convex combinations including the AT value.

Next, we show the logical independence of the six axioms of Theorem 3.8.

1. The conjunctive permission value satisfies efficiency, linearity, the inessential player property, interior unanimity symmetry, and predecessor necessity. It does not satisfy 
collusion neutrality.

2. The solution $f(N, v, D)=\operatorname{AT}\left(N, v, L_{D}\right)$ that applies the Average Tree value to the original game $v$ on the associated undirected graph $L_{D}$ satisfies efficiency, linearity, the inessential player property, interior unanimity symmetry, and collusion neutrality. It does not satisfy predecessor necessity.

3. The solution that assigns all worth $v(N)$ to the top node and zero to all other players satisfies efficiency, linearity, the inessential player property, predecessor necessity, and collusion neutrality. It does not satisfy interior unanimity symmetry.

4. The equal division solution given by $f_{i}(N, v, D)=v(N) /|N|$ for all $i \in N$ satisfies efficiency, linearity, interior unanimity symmetry, predecessor necessity, and collusion neutrality. It does not satisfy the inessential player property.

5. For $(N, v, D) \in \mathcal{G}_{\mathcal{T}}^{N}$, let $(N, \bar{v}) \in \mathcal{G}^{N}$ be given by $\bar{v}=v(N) u^{N \backslash I(N, v, D)}$, where $I(N, v, D)$ is the set of inessential players in $(N, v, D)$. The solution $\bar{f}(N, v, D)=$ $\psi(N, \bar{v}, D)$ satisfies efficiency, the inessential player property, interior unanimity symmetry, predecessor necessity, and collusion neutrality. It does not satisfy linearity.

6. The zero solution given by $f_{i}(N, v, D)=0$ for all $i \in N$ satisfies linearity, the inessential player property, interior unanimity symmetry, predecessor necessity, and collusion neutrality. It does not satisfy efficiency.

We end this section by mentioning that in Theorem 3.8 predecessor necessity can be replaced by independence of irrelevant coalitions as used by van den Brink, Katsev, and van der Laan (2011) for games on union closed systems, stating that the payoff only depends on the worths of feasible coalitions. A similar axiom, called connectedness, is used by van den Brink, van der Laan, and Pruzhansky (2011) for communication graph games, saying that the payoffs only depend on the worths of connected coalitions.

\section{An axiomatization using fairness}

In this section we characterize the AT permission value by modifying the component fairness Axiom 2.1 to the framework of permission tree games.

We say that in a permission tree game $(N, v, D) \in \mathcal{G}_{\mathcal{T}}^{N}$ some player $i \in N$ is enforcing power over player $j \in N$ when $i$ vetoes any coalition that contains $j$ or any of its subordinates but does not contain player $i$. Since $i$ is a predecessor of $j$, and thus is a superior of every player in cone $K_{j}^{i j}$, any $h \in K_{j}^{i j}$ has $j$ as one of its superiors and thus also needs permission of $i$. So, the players in $K_{j}^{i j}$ cannot cooperate without permission of 
player $i$. It follows that $\sigma_{D}\left(K_{j}^{i j}\right)=\emptyset$, i.e., in the permission structure $(N, D)$ the players in $K_{j}^{i j}$ earn worth zero without permission from player $i$. On the other hand, neither player $i$ nor any of the predecessors of player $i$ can force the players in $K_{j}^{i j}$ to cooperate. The corresponding game in which the players in $\{j\} \cup \widehat{S}_{D}(j)$ are not cooperating is the game $\left(N, v^{-i j}\right) \in \mathcal{G}^{N}$ given by $v^{-i j}(T)=v\left(T \backslash\left(\{j\} \cup \widehat{S}_{D}(j)\right)\right)$ for all $T \in 2^{N}$.

Applying a similar idea as component fairness, but now with respect to the enforcement of permission power, we obtain the following axiom.

\section{Axiom 4.1 Permission Component Fairness}

A solution $f$ on the class $\mathcal{G}_{\mathcal{T}}^{N}$ of permission tree games satisfies permission component fairness if, for every $(N, v, D) \in \mathcal{G}_{\mathcal{T}}^{N}$ and for any link $(i, j) \in D$, it holds that

$$
\frac{\sum_{h \in K_{i}^{i j}}\left[f_{h}(N, v, D)-f_{h}\left(N, v^{-i j}, D\right)\right]}{\left|K_{i}^{i j}\right|}=\frac{\sum_{h \in K_{j}^{i j}}\left[f_{h}(N, v, D)-f_{h}\left(N, v^{-i j}, D\right)\right]}{\left|K_{j}^{i j}\right|} .
$$

The following theorem characterizes the AT permission value by efficiency, the inessential player property, and permission component fairness.

Theorem 4.2 On the class $\mathcal{G}_{\mathcal{T}}^{N}$ of permission tree games, the AT permission value is the unique solution that satisfies efficiency, the inessential player property, and permission component fairness.

Proof. It follows from Theorem 3.8 that the AT permission value satisfies efficiency and the inessential player property. To prove that the AT permission value satisfies permission component fairness, take any $\operatorname{arc}(i, j) \in D$. Then all players in $K_{j}^{i j}$ are inessential in $\left(N, v^{-i j}, D\right)$, so

$$
\sum_{h \in K_{j}^{i j}} \psi_{h}\left(N, v^{-i j}, D\right)=0
$$

Since the AT permission value is efficient, we have that

$$
\sum_{h \in K_{i}^{i j}} \psi_{h}\left(N, v^{-i j}, D\right)=v^{-i j}(N)-\sum_{h \in K_{j}^{i j}} \psi_{h}\left(N, v^{-i j}, D\right)=v\left(K_{i}^{i j}\right) .
$$

For permission component fairness to hold, we therefore have to show that

$$
\frac{\sum_{h \in K_{j}^{i j}} \psi_{h}(N, v, D)}{\left|K_{j}^{i j}\right|}=\frac{\sum_{h \in K_{i}^{i j}} \psi_{h}(N, v, D)-v\left(K_{i}^{i j}\right)}{\left|K_{i}^{i j}\right|} .
$$

Recall that the AT permission value $\psi$ is defined as the AT value applied to the cycle-free graph game $\left(N, v_{D}, L_{D}\right)$, so as the average of the $|N|$ hierarchical outcomes of the game 
$\left(N, v_{D}\right)$ on the graph $\left(N, L_{D}\right)$, each one associated to precisely one of the players. The hierarchical outcome associated with player $k \in N$ gives to player $\ell \in N$ payoff

$$
t_{\ell}^{k}\left(N, v_{D}, L_{D}\right)=v_{D}\left(C_{k}^{L_{D}}(\ell)\right)-\sum_{h \in C_{k}^{L_{D}}(\ell) \cap N^{L_{D}(\ell)}} v_{D}\left(C_{k}^{L_{D}}(h)\right), \quad \ell \in N .
$$

Consider any arc $(i, j) \in D$. When $k \in K_{i}^{i j}$, then $C_{k}^{L_{D}}(j)=K_{j}^{i j}$ and so the total payoff at vector $t^{k}\left(N, v_{D}, L_{D}\right)$ to the players in $K_{j}^{i j}$ is equal to $v_{D}\left(K_{j}^{i j}\right)$. Since $v_{D}\left(K_{j}^{i j}\right)=0$, it follows that the players in $K_{j}^{i j}$ get total payoff equal to zero in $\left|K_{i}^{i j}\right|$ of the $|N|$ hierarchical outcomes. When $k \in K_{j}^{i j}$, then $C_{k}^{L_{D}}(i)=K_{i}^{i j}$ and so the total payoff at vector $t^{k}\left(N, v_{D}, L_{D}\right)$ to the players in $K_{i}^{i j}$ is equal to $v_{D}\left(K_{i}^{i j}\right)=v\left(K_{i}^{i j}\right)$. From efficiency it follows that the players in $K_{j}^{i j}$ get total payoff equal to $v(N)-v\left(K_{i}^{i j}\right)$. This occurs in $\left|K_{j}^{i j}\right|$ of the $|N|$ hierarchical outcomes. It follows that

$$
\sum_{h \in K_{j}^{i j}} \psi_{h}(N, v, D)=\frac{\left|K_{j}^{i j}\right|\left(v(N)-v\left(K_{i}^{i j}\right)\right)}{|N|} .
$$

From the reasoning above it also follows that the players in $K_{i}^{i j}$ get total payoff equal to $v\left(K_{i}^{i j}\right)$ in the $\left|K_{j}^{i j}\right|$ of the $|N|$ hierarchical outcomes where $k \in K_{j}^{i j}$, and they get total payoff $v(N)$ in the $\left|K_{i}^{i j}\right|$ of the $|N|$ hierarchical outcomes where $k \in K_{i}^{i j}$. So,

$$
\sum_{h \in K_{i}^{i j}} \psi_{h}(N, v, D)=\frac{\left|K_{j}^{i j}\right| v\left(K_{i}^{i j}\right)+\left|K_{i}^{i j}\right| v(N)}{|N|}
$$

Substituting $\left|K_{j}^{i j}\right|=|N|-\left|K_{i}^{i j}\right|$ in the latter equation yields

$$
\sum_{h \in K_{i}^{i j}} \psi_{h}(N, v, D)-v\left(K_{i}^{i j}\right)=\frac{\left|K_{i}^{i j}\right|\left(v(N)-v\left(K_{i}^{i j}\right)\right)}{|N|} .
$$

From equations (4.3) and (4.4) it follows that $\psi$ satisfies permission component fairness.

It remains to show that the three axioms characterize a unique solution. Let $f$ be a solution satisfying the three axioms. Then efficiency requires that

$$
\sum_{h \in N} f_{h}(N, v, D)=v(N)
$$

and permission component fairness requires

$$
\frac{\sum_{h \in K_{i}^{i j}}\left[f_{h}(N, v, D)-f_{h}\left(N, v^{-i j}, D\right)\right]}{\left|K_{i}^{i j}\right|}=\frac{\sum_{h \in K_{j}^{i j}}\left[f_{h}(N, v, D)-f_{h}\left(N, v^{-i j}, D\right)\right]}{\left|K_{j}^{i j}\right|}, \quad(i, j) \in D .
$$

All players in $K_{j}^{i j}$ are inessential in $\left(N, v^{-i j}, D\right)$, so by the inessential player property

$$
\sum_{h \in K_{j}^{i j}} f_{h}\left(N, v^{-i j}, D\right)=0, \quad(i, j) \in D .
$$


Since $f$ is efficient, we have that

$$
\sum_{h \in K_{i}^{i j}} f_{h}\left(N, v^{-i j}, D\right)=v^{-i j}(N)-\sum_{h \in K_{j}^{i j}} f_{h}\left(N, v^{-i j}, D\right)=v\left(K_{i}^{i j}\right), \quad(i, j) \in D .
$$

We find that

$$
\frac{\sum_{h \in K_{j}^{i j}} f_{h}(N, v, D)}{\left|K_{j}^{i j}\right|}=\frac{\sum_{h \in K_{i}^{i j}} f_{h}(N, v, D)-v\left(K_{i}^{i j}\right)}{\left|K_{i}^{i j}\right|}, \quad(i, j) \in D .
$$

Since $(N, D) \in \mathcal{D}_{\mathcal{T}}^{N}$, the number of $\operatorname{arcs}$ in $D$ is equal to $|N|-1$. So, the total number of equations in (4.5) and (4.6) is $|N|$. Since all $|N|$ equations are linearly independent, the system in (4.5) and (4.6) has a unique solution in the $|N|$ variables $f_{h}(N, v, D), h \in N$.

The three axioms of Theorem 4.2 are logically independent. The equal division solution satisfies efficiency and permission component fairness, but not the inessential player property. The conjunctive permission value satisfies efficiency and the inessential player property, but not permission component fairness. Finally, the zero solution satisfies the inessential player property and permission component fairness, but not efficiency.

\section{Comparison with the conjunctive permission value}

In this section we compare the Average Tree permission value with the conjunctive permission value defined in Subsection 2.3. As shown in the first case under logical independence in Section 3, the conjunctive permission value satisfies all axioms of Theorem 3.8 except collusion neutrality. It turns out that strengthening interior unanimity symmetry by requiring that all players in $T$ get the same payoff in $\left(N, u^{T}, D\right)$, and not only the interior players, we can delete collusion neutrality to obtain an axiomatization of the conjunctive permission value.

\section{Axiom 5.1 Unanimity symmetry}

For every $T \in 2^{N} \backslash\{\emptyset\},\left(N, u^{T}, D\right) \in \mathcal{G}_{\mathcal{T}}^{N}$, and $i, j \in T$ it holds that $f_{i}\left(N, u^{T}, D\right)=$ $f_{j}\left(N, u^{T}, D\right)$.

Theorem 5.2 On the class $\mathcal{G}_{\mathcal{T}}^{N}$ of permission tree games the conjunctive permission value is the unique solution that satisfies efficiency, linearity, the inessential player property, unanimity symmetry, and predecessor necessity.

Proof. It is known from van den Brink and Gilles (1996) that the conjunctive permission value satisfies efficiency, linearity, and the inessential player property. Unanimity symmetry 
follows from symmetry ${ }^{7}$ of the Shapley value and the fact that $i, j \in T$ are symmetric players in $\left(N, u_{D}^{T}\right)=\left(N, u^{\widehat{P}_{D}(T)}\right)$. Predecessor necessity follows since $u_{D}^{T}=u_{D}^{T \cup\left\{p_{D}(j)\right\}}$ for all $T \subset N$ and $j \in T$.

We are left to show uniqueness. Suppose that $f$ satisfies the five axioms and consider the unanimity game $\left(N, u^{T}, D\right), T \in \Omega_{D}$. Similar as in the proof of Theorem 3.8, since $T$ is feasible, for every $i \notin T$ it holds that also all subordinates of $i$ are not in $T$ and so $i$ is inessential in $\left(N, u^{T}, D\right)$. Thus $f_{i}\left(N, u^{T}, D\right)=0$ by the inessential player property. From unanimity symmetry it follows that all players in $T$ have equal payoff. These payoffs then follow from efficiency. If $T \notin \Omega_{D}$ then, similar as in the proof of Theorem 3.8, by predecessor necessity we have that $f\left(N, u^{T}, D\right)=f\left(N, u^{F(T)}, D\right)$. Hence, for every unanimity game the payoffs are uniquely determined by efficiency, the inessential player property, unanimity symmetry, and predecessor necessity. Finally, $f(N, v, D)$ is uniquely determined by linearity for every $(N, v, D) \in \mathcal{G}_{\mathcal{T}}^{N}$.

Theorems 3.8 and 5.2 show an important difference between the conjunctive permission value and AT permission value. By unanimity symmetry, in a unanimity game the conjunctive permission value treats all the players in $T$ the same. Similar to the AT value for cycle-free communication graph games, in the AT permission value the players in $T$ who have neighbors outside $T$ have some 'responsibility' or 'representability' for these players. Therefore, the interior players are treated equally, but the other players in $T$, i.e., the 'boundary' players, earn a payoff that depends on the substructure where they are the top player and that contains them and their subordinates. This is taken care for by collusion neutrality which, at each step, assigns some 'joint payoff' to a player and one of its successors when they collude.

The next five solutions show that the five axioms of Theorem 5.2 are logically independent. For each solution we state the four axioms satisfied by the solution, consequently it does not satisfy the fifth axiom. The solution $f_{i}(N, v, D)=\operatorname{My}\left(N, v, L_{D}\right)$ satisfies efficiency, linearity, the inessential player property, and unanimity symmetry. The AT permission value satisfies efficiency, linearity, the inessential player property, and predecessor necessity. The equal division solution satisfies efficiency, linearity, unanimity symmetry, and predecessor necessity. The solution $\bar{f}$ defined at the end of Section 3 (as fifth alternative solution showing logical independence of the axioms in Theorem 3.8) satisfies efficiency, the inessential player property, unanimity symmetry, and predecessor necessity. Finally, the zero solution given by $f_{i}(N, v, D)=0$ for all $i \in N$ satisfies linearity, the inessential player property, unanimity symmetry, and predecessor necessity.

\footnotetext{
${ }^{7} \mathrm{~A}$ TU game solution $f$ is symmetric if for every TU game $(N, v)$ and every pair $i, j \in N$ such that $v(S \cup\{i\})=v(S \cup\{j\})$ for every $S \in 2^{N \backslash\{i, j\}}$, it holds that $f_{i}(N, v)=f_{j}(N, v)$.
} 
Next, we modify permission component fairness to get an axiomatization of the conjunctive permission value. Similar as in Section 4, consider an $\operatorname{arc}(i, j) \in D$ and suppose that player $i$ is enforcing its power over $j$ in the sense that it does not allow player $j$ and all its subordinates to act. Then player $j$ and all its subordinates, i.e., the players in $K_{j}^{i j}$, become null players. On the other hand, the players in $K_{j}^{i j}$ can refuse to cooperate with the players in $K_{i}^{i j}$. Applying a similar idea as Myerson's fairness, but now with respect to the enforcement of permission power, we obtain the following axiom.

\section{Axiom 5.3 Permission Fairness}

A solution $f$ on the class $\mathcal{G}_{\mathcal{T}}^{N}$ of permission tree games satisfies permission fairness if, for every $(N, v, D) \in \mathcal{G}_{\mathcal{T}}^{N}$ and for any pair $i, j \in N$ with $(i, j) \in D$, it holds that

$$
f_{i}(N, v, D)-f_{i}\left(N, v^{-i j}, D\right)=f_{j}(N, v, D)-f_{j}\left(N, v^{-i j}, D\right) .
$$

Replacing in Theorem 4.2 permission component fairness by this permission fairness characterizes the conjunctive permission value.

Theorem 5.4 On the class $\mathcal{G}_{\mathcal{T}}^{N}$ of permission tree games, the conjunctive permission value is the unique solution that satisfies efficiency, the inessential player property, and permission fairness.

Proof. It is known that the conjunctive permission value $\varphi$ satisfies efficiency and the inessential player property. To show permission fairness, consider $(N, v, D) \in \mathcal{G}_{\mathcal{T}}^{N}$ and $i, j \in N$ with $(i, j) \in D$. According to Proposition 2.3 in van den Brink and Gilles (1996), it follows by applying the dividend formula of the Shapley value that

$$
\varphi_{i}(N, v, D)=\sum_{\left\{T \in 2^{N} \mid i \in F(T)\right\}} \Delta_{v}(T) /|F(T)|, \quad i \in N .
$$

We therefore have that

$$
\begin{aligned}
\varphi_{i}(N, v, D)-\varphi_{i}\left(N, v^{-i j}, D\right) & =\sum_{\substack{T \in 2^{N} \\
i \in F(T)}} \frac{\Delta_{v}(T)}{|F(T)|}-\sum_{\substack{T \in 2^{N} \\
i \in F(T)}} \frac{\Delta_{v}-i j(T)}{|F(T)|} \\
& =\sum_{\substack{T \in 2^{N} \\
i \in F(T)}} \frac{\Delta_{v}(T)}{|F(T)|}-\sum_{\substack{i \in F(T), j \notin F(T) \\
T(T) 2^{N}}} \frac{\Delta_{v}(T)}{|F(T)|} \\
& =\sum_{\substack{T \in 2^{T} N \\
\{i, j \subseteq F(T)}} \frac{\Delta_{v}(T)}{|F(T)|}=\sum_{\substack{T \in 2^{N} \\
j \in F(T)}} \frac{\Delta_{v}(T)}{|F(T)|}=\varphi_{j}(N, v, D),
\end{aligned}
$$

where the second equality follows since $\Delta_{v^{-i j}}(T)=\Delta_{v}(T)$ if $j \notin F(T)$, and $\Delta_{v^{-i j}}(T)=0$ otherwise, and the fourth equality follows since $j \in F(T)$ implies that $i \in F(T)$ for all $T \in 2^{N}$. Since $\varphi_{j}\left(N, v^{-i j}, D\right)=0$ by the inessential player property, it follows that $\varphi$ satisfies permission fairness.

We show uniqueness by induction on the cardinality of the set $I(N, v, D)$ of inessential players. Let $(N, v, D) \in \mathcal{G}_{\mathcal{T}}^{N}$ and $i_{0} \in N$ be the top player in $(N, D)$. If $|I(N, v, D)|=$ 
$|N|$, i.e., all players are inessential, then the inessential player property implies that $f_{i}(N, v, D)=0$ for all $i \in N$. If $|I(N, v, D)|=|N|-1$ then all players in $N \backslash\left\{i_{0}\right\}$ are inessential players, and thus the inessential player property implies that $f_{i}(N, v, D)=0$ for all $i \in N \backslash\left\{i_{0}\right\}$. Efficiency then determines that $f_{i_{0}}(N, v, D)=v(N)$.

Proceeding by induction, assume that $f\left(N, v^{\prime}, D\right)$ is determined when $|I(N, v, D)|=$ $k, 1 \leq k \leq|N|-1$, and suppose that $|I(N, v, D)|=k-1$. The inessential player property implies that $f_{i}(N, v, D)=0$ for all $i \in I(N, v, D)$. For every $i \in N \backslash\left(I(N, v, D) \cup\left\{i_{0}\right\}\right)$ and $j=p_{D}(i)$, permission fairness requires that

$$
f_{i}(N, v, D)-f_{i}\left(N, v^{-i j}, D\right)=f_{j}(N, v, D)-f_{j}\left(N, v^{-i j}, D\right) .
$$

Since the payoffs $f_{i}\left(N, v^{-i j}, D\right)$ and $f_{j}\left(N, v^{-i j}, D\right)$ are determined by the induction hypothesis, and $(N, D)$ is an oriented tree this yields $|N|-|I(N, v, D)|-1$ linear equations. Together with efficiency

$$
\sum_{h \in N} f_{h}(N, v, D)=v(N)
$$

the total number of equations is $|N|-|I(N, v, D)|$. Since these equations are linearly independent, the system of $|N|-|I(N, v, D)|$ equations (5.7) and (5.8) has a unique solution in the $|N|-|I(N, v, D)|$ variables $f_{h}(N, v, D), h \in N \backslash I(N, v, D)$.

Note that, whereas in the uniqueness part of the proof of Theorem 4.2, for every arc in the permission structure permission component fairness yields a non-trivial equation resulting in $|N|-1$ linear independent equations, in the proof of Theorem 5.4 permission fairness does not give a new equation like (5.7) if $i$ is an inessential player since $v^{-i j}=v$ (with $j=p_{D}(i)$ ) in that case. But using the inessential player property to fix the payoffs of the inessential players, permission fairness gives $|N|-|I(N, v, D)|-1$ linear independent equations which, together with efficiency, determine the payoffs of the other players.

The three axioms of Theorem 5.4 are logically independent. The equal division solution satisfies efficiency and permission fairness, but not the inessential player property. The AT permission value satisfies efficiency and the inessential player property, but not permission fairness. Finally, the zero solution satisfies the inessential player property and permission fairness, but not efficiency.

We finally remark that the conjunctive permission value does not satisfy fairness in the sense that deleting an arc yields the same change in payoff for the two players incident with that arc, even on classes where it is allowed to delete arcs. ${ }^{8}$ As shown in van den Brink (1997) this does hold for the disjunctive permission value when deleting an arc

\footnotetext{
${ }^{8}$ Note that, considering $D$ as a permission structure, deleting arc $(i, j) \in D$ has as effect that $j$ can cooperate with all feasible coalitions within $K_{j}^{i j}$.
} 
where the successor has at least two predecessors but not when the successor has only one predecessor. Therefore this fairness axiom 'has no bite' for permission tree games. This shows an important difference between stating fairness axioms in terms of deleting arcs or enforcing power. Also, we can extend the AT permission value to more general permission structures by applying the (arc) fairness properties used in van den Brink (1997).

We end this section with an example illustrating some differences between the conjunctive permission value and the AT permission value.

Example 5.5 Consider the game $(N, v, D)$ with player set $N=\{1,2,3,4,5\}$, characteristic function $v=u^{\{2,4\}}$ and permission structure $D=\{(1,2),(1,3),(3,4),(3,5)\}$. The conjunctive permission value assigns payoff vector $\varphi(N, v, D)=(1 / 4,1 / 4,1 / 4,1 / 4,0)$ and the AT permission value $\psi(N, v, D)=(1 / 5,1 / 5,1 / 5,2 / 5,0)$. By the inessential player property, in both payoff vectors player 5 has payoff zero. By unanimity symmetry, in the conjunctive permission value all other players earn $1 / 4$. This is not the case for the AT permission value payoffs. By interior unanimity symmetry the players 1,2 , and 4 earn the same. By collusion neutrality, players 3 and 5 together earn the same as in $\psi\left(N, u^{N}, D\right)=(1 / 5,1 / 5,1 / 5,1 / 5,1 / 5)$. Since $\psi_{5}(N, v, D)=0$, we obtain $\psi_{3}(N, v, D)=$ $2 / 5$ and so $\psi_{1}(N, v, D)=\psi_{2}(N, v, D)=\psi_{4}(N, v, D)=1 / 5$.

\section{Concluding remarks}

In this paper we have studied games with an oriented tree as permission structure. Since in such games players can only generate a surplus if they get permission to collaborate from all their superiors, only coalitions containing the superiors of all players involved in the coalition can form. In van den Brink and Gilles (1996) the conjunctive permission value for this class of games is introduced.

We show that the axioms of efficiency, linearity, the inessential player property, predecessor necessity, interior unanimity symmetry, and collusion neutrality characterize a unique value, the AT permission value. The AT permission value can also be axiomatized by efficiency, the inessential player property, and permission component fairness. The AT permission value can be easily computed as the average of $|N|$ hierarchical outcomes, where $|N|$ is the number of players. Each hierarchical outcome is an $|N|$-dimensional payoff vector, whose values can be found by the evaluation of $|N|$ simple and explicit linear expressions.

We also evaluate the connection between the AT permission value and the conjunctive permission value. If we strengthen the interior unanimity symmetry axiom to unanimity symmetry and drop the collusion neutrality axiom, we obtain a new axiomatization for the conjunctive permission value. When we replace permission component 
fairness by permission fairness, we obtain another new axiomatization of the conjunctive permission value.

Several games in the economic literature turn out to be the conjunctive restricted game on an appropriate digraph, such as the auction games of Graham, Marshall and Richard (1990), the so-called DR-polluted river game of Ni and Wang (2007) and its generalization in Dong, Ni and Wang (2012), and the dual of the airport game of Littlechild and Owen (1973), see also Brânzei, Fragnelli and Tijs (2002). These papers study the Shapley value of the corresponding game which is a special case of the conjunctive permission value. As an alternative the AT permission value can be studied for these applications.

\section{References}

Algaba, E., J.M. Bilbao, R. van den Brink, And A. Jiménez-Losada (2004), "Cooperative Games on Antimatroids", Discrete Mathematics, 282, 1-15.

BÉal, S., A. Lardon, E. Rémila, and P. Solal (2012), "The Average Tree Solution for Multi-Choice Forest Games", Annals of Operations Research, 196, 27-51.

BÉal, S., E. RÉmila, and P. Solal (2010), "Rooted Tree Solutions for Tree Games", European Journal of Operational Research, 203, 404-408.

BilbaO, J.M. (2000), Cooperative Games on Combinatorial Structures, Kluwer Academic Publishers, Dordrecht.

Borm P., G. Owen, And S. Tijs (1992), "On the Position Value for Communication Situations", SIAM Journal on Discrete Mathematics, 5, 305-320.

Brânzei, R., V. Fragnelli, and S. TiJs (2002), "Tree-Connected Peer Group Situations and Peer Group Games", Mathematical Methods of Operations Research, 55, 93-106.

Brink, R. van Den (1997), "An Axiomatization of the Disjunctive Permission Value for Games with a Permission Structure", International Journal of Game Theory, 26, 27-43.

BRINK, R. VAN DEN (2012) "Efficiency and Collusion Neutrality in Cooperative Games and Networks", Games and Economic Behavior, 76, 344-348.

Brink, R. van Den, AND R.P. Gilles (1996), "Axiomatizations of the Conjunctive Permission Value for Games with Permission Structures", Games and Economic Behavior, 12, 113-126.

Brink, R. van den, I. Katsev, and G. van der LaAn (2011), "Axiomatizations of Two Types of Shapley Values for Games on Union Closed Systems", Economic Theory, 47, 175-188.

Brink, R. van den, G. van der LaAn, And V. Pruzhansky (2011), "Harsanyi Power Solutions for Graph-restricted Games", International Journal of Game Theory, 40, 87-110.

Demange, G. (2004), "On Group Stability in Hierarchies and Networks", Journal of Political Economy, 112, 754-778.

Dilworth, R.P. (1940), "Lattices with Unique Irreducible Decompositions", Annals of Mathematics, 41, $771-777$. 
Dong, B., D. Ni, ANd Y. Wang (2012), "Sharing a Polluted River Network", Environmental and Resource Economics, 53, 367-387.

Edelman, P.H., And R.E. Jamison (1985), "The Theory of Convex Geometries", Geometrica Dedicata, 19, 247-270.

Faigle, U., And W. Kern (1992), "The Shapley Value for Cooperative Games under Precedence Constraints", International Journal of Game Theory, 21, 249-266.

Gilles, R.P., And G. Owen (1994), "Cooperative Games and Disjunctive Permission Structures", Department of Economics, Virginia Polytechnic Institute and State University, Blacksburg, Virginia.

Gilles R.P., G. Owen, and R. van den Brink (1992), "Games with Permission Structures: The Conjunctive Approach", International Journal of Game Theory, 20, 277-293.

Graham, D.A., R.C. Marshall, And J.F. Richard (1990), "Differential Payments within a Bidder Coalition and the Shapley Value", American Economic Review, 80, 493-510.

Haller, H. (1994), "Collusion Properties of Values", International Journal of Game Theory, 23, 261-281.

Harsanyi, J.C. (1959), "A Bargaining Model for Cooperative n-Person Games", in Contributions to the Theory of Games II (Annals of Mathematics Studies 40), A.W. Tucker and R.D Luce (eds.), Princeton University Press, Princeton, pp. 325-355.

Herings, P.J.J., G. van der LaAn, and A.J.J. Talman (2008), "The Average Tree Solution for CycleFree Graph Games", Games and Economic Behavior, 62, 77-92.

Li, L., AND X. Li (2011), "The Covering Values for Acyclic Digraph Games", International Journal of Game Theory, 40, 697-718.

Littlechild, S.C., And G. Owen (1973), "A Simple Expression for the Shapley Value in a Special Case", Management Science, 20, 370-372.

Myerson, R.B. (1977), "Graphs and Cooperation in Games", Mathematics of Operations Research, 2, 225-229.

Ni, D., And Y. Wang (2007), "Sharing a Polluted River", Games and Economic Behavior, 60, 176-186.

Shapley, L.S. (1953) "A Value for $n$-Person Games", in Contributions to the Theory of Games II (Annals of Mathematics Studies 28), H.W. Kuhn and A.W. Tucker (eds.), Princeton University Press, Princeton, pp. 307-317. 